\title{
RACIONALIDAD Y CONTROL EN LAS ORGANIZACIONES COMPLEJAS *
}

José Pérez Vilariño y Richard A. Schoenherr

El análisis de los principales procesos organizativos constituye una de las vías más practicables de acceso a los procesos sociales básicos. «La organización - escribe Lucien Karpik - es la realidad social que mejor expresa y permite identificar las reglas de funcionamiento y evolución de los procesos sociales» (L. Karpik, 1978: 17).

La proliferación de las organizaciones durante los últimos cincuenta años es tal vez el factor más significativo de cambio social, que - según la reciente expresión de J. Coleman- está dando origen a una «sociedad asimétrica», en la que los individuos no pueden defender inmediatamente sus intereses (J. Coleman, 1983).

Los conceptos de racionalidad y de control organizativos serán los hilos conductores de estas páginas. La tarea que se asignan es la de desenredar la maraña a que han dado lugar sus formas históricas de entrecruzarse, buscando al mismo tiempo un patrón teórico que permita entretejerlos de nuevo sistemáticamente.

El análisis avanzará desde los modelos más simples de racionalidad y ejercicio abierto del poder, con formas directas de control, hasta sus variantes

* Los autores agradecen la valiosa y desinteresada colaboración de Celia Muñoz Goy, que ha facilitado la sistematización de la bibliografía y la redacción final del texto. También nuestra gratitud a Ana García Filgueira por su lucha incansable frente a nuestros garabatos. 
más complejas que recurren a mecanismos imperceptibles de dominación -unobtrusive control.

La atención preferente al proceso de control responde a la idea -de inspiración weberiana y cada vez más generalizada - de que la esencia de las organizaciones es el poder.

Hasta hace poco, estos fenómenos eran predominantemente analizados desde la reduccionista óptica del taylorismo. La vinculación a Weber se reducía a la descripción del tipo ideal de burocracia, olvidando las cuestiones centrales de la legitimación y la dominación, así como su exigencia de una metodología libre de implicaciones interesadas o valorativas.

El análisis de los procesos organizativos, a la luz de los clásicos, permite estudiar la naturaleza de las organizaciones, sin limitarse únicamente a su empleo como técnicas de control empresarial.

Por su parte, la introducción en el campo de las organizaciones del concepto de entorno, tomado de la ecología, permitirá comprender la creciente importancia de la interacción entre los procesos económicos y políticos.

\section{PROCESOS SOCIALES Y PROCESOS ORGANIZATIVOS}

El paradigma parsoniano de los cuatro requisitos funcionales sintetiza los procesos sociales y organizativos básicos. El primero define los procesos de producción económica, que aseguran los recursos necesarios al sistema mediante su adaptación al entorno. El segundo da origen a procesos políticos, mediante los cuales la organización y la sociedad establecen sus objetivos. Ambos procesos son de carácter instrumental y se definen por su nivel de eficiencia en la realización de sus tareas específicas: la obtención y la asignación de los recursos necesarios.

Los otros dos procesos se refieren al funcionamiento interno del sistema y buscan su estabilidad a través de la integración social y el mantenimiento de los valores culturales.

Diferentes perspectivas analíticas han prestado atención a uno o dos de estos procesos, pero ninguna ha estudiado sistemáticamente los cuatro.

Los modelos económicos de análisis de la organización tienen como objetivo la búsqueda de la mayor eficiencia en el ajuste de los medios a unos fines que se suponen dados.

Para Taylor y la Escuela de la Organización Científica del Trabajo, así como para Fayol y la Escuela de la Administración Científica, el problema de la organización se reducía en el fondo a una cuestión de control, esto es, «a conseguir un mecanismo general realmente efectivo para hacer rendir al trabajador» (Hobsbawn, 1975: 221). La organización se concibe, en última instancia, como una máquina (Macbine Theory) con una unidad de mando y una autoridad jerarquizada. 
En el campo más específicamente sociológico, esta racionalidad de carácter económico - a partir del tipo ideal de burocracia de Weber y de la concepción reificante de los hechos sociales de Durkheim- llevó al predominio del modelo racional-estructural, presentado, entre otros, por Aiken y Hage, Schoenherr y Blau, Pugh y la Escuela de Aston. Dentro de esta perspectiva puede incluirse también la primera formulación del modelo de March y Simon.

Las críticas a este modelo racional destacan su perspectiva estática y consensual. Aparte del olvido de las motivaciones, no tiene en cuenta los conflictos de poder y la influencia del entorno, que limitan la racionalidad del individuo y de las organizaciones.

El debate sobre los objetivos es lo que caracteriza la perspectiva politica. Muy pocos modelos se fijan exclusivamente en los procesos políticos de las organizaciones. Por el contrario, la atención simultánea a los procesos políticos y económicos, que supone una vuelta a Marx y Max Weber, constituye hoy la corriente predominante denominada Economía Política de las Organizaciones.

Los elementos del grupo no marxista son la fundamentación del poder en el control de la incertidumbre (Crozier) y la formación de coaliciones (Cyert y March, 1963), que ponen en cuestión los propios fines de la organización. Por otra parte, la atención primordial al entorno (Zald, Aldrich) obliga a considerar la economía política interna de la organización en interacción con otra economía política externa.

Los autores de inspiración marxista intentan ir más allá de la óptica de los managers. Su atención primordial se centra en el proceso laboral, realizando sobre todo análisis históricos que intentan descubrir los cambios en las formas de dominación a través de las organizaciones. Los análisis de Hymer sobre las organizaciones multinacionales son tal vez los más representativos de esta corriente.

La perspectiva derivada de los procesos de interacción social dio entrada a otros factores distintos de los económicos y políticos. Los modelos representativos de esta orientación responden a la Escuela de las Relaciones Humanas.

La variable clave para estos autores es el estilo de supervisión y de liderazgo que determinan el tipo de control. De aquí deriva toda una literatura sobre técnicas de relaciones humanas, dinámica de grupos, liderazgo, etc., actualmente percibida con una fuerte carga ideológica y que responde al propósito de despolitizar la organización. Los modelos de las relaciones humanas ignoran sistemáticamente los procesos económicos y políticos, dando por supuesto que una mejor integración social sería capaz de resolver todos los problemas organizativos.

Además de Elton Mayo destacan Argyris y el japonés Ouchi. Estas escuelas no perciben la diferencia entre las relaciones interpersonales y las relaciones sociales de producción en las que se insertan. Su objetivo es, en defi- 
nitiva, el mismo que el de la organización científica del trabajo: conseguir un control efectivo, si bien la racionalidad empleada es diferente. Se trata de una forma de control menos directo y que reposa, en última instancia, en los valores culturales.

«No deja de ser una paradoja que [las Relaciones Humanas] vengan a terminar en un determinismo tan mecanicista como el de los clásicos», porque, si bien «las relaciones humanas son más naturales que la "dirección científica"..., persiguen el mismo fin: la eficacia» (S. del Campo, 1962: 62 y 109).

Por último, los modelos centrados en los procesos latentes de mantenimiento de los valores culturales son los más recientes. Como señalan March y Simon (1969: 205), «los aspectos cognitivos de la conducta organizativa son, todavía hoy, terrenos casi sin explorar».

Los estudios de Weber sobre la interacción entre cultura y economía, o sobre las formas de institucionalización de los valores, anticipan la base teórica de estos modelos, que remiten al concepto de construcción social de la realidad de Berger y Luckmann. Las organizaciones son externalizaciones y objetivaciones, esto es, formas concretas culturalmente definidas, de construcciones sociales. A medida que la sociedad se hace más compleja se va convirtiendo en una red más amplia de tales construcciones, las cuales, a su vez, procesan o construyen realidades sociales de acuerdo con sus valores y de un modo similar al descrito por la teoría de la etiqueta.

La atención a la cultura organizativa es la forma más asequible de descubrir los procesos latentes de control. La cultura organizativa define los valores a los que tienen que ajustarse las metas operativas, ofrece los instrumentos para la movilización de los miembros y define el uso legítimo de los recursos. $\mathrm{Y}$ todo esto lo realiza desde dentro del propio individuo-miembro, que ha interiorizado la cultura de la organización, sin apenas necesidad de recurrir a controles directos. "Lo mismo que los estados-nación y las sociedades primitivas -escribe Zald-, también las organizaciones complejas tienen constituciones... La constitución de una organización es su estructura normativa fundamental... Es un conjunto de acuerdos y presupuestos tácitos que definen los límites y los objetivos del grupo (o colectividad), así como las responsabilidades y derechos de los participantes» (Zald, 1981: 240).

Tanto Zald como Hirschman subrayan la interrelación entre los procesos organizativos de carácter económico, político y cultural. Hirschman (1970) elabora un brillante y parsimonioso modelo. En su conocida obra -Exit, Voice and Loyalty - analiza las relaciones entre las tasas de abandono - exitde la organización (que responden al cálculo de carácter económico del costebeneficio de seguir o no participando), el nivel de participación activa - voice- (que constituye una presión política) y la lealtad - loyalty - (que deriva de las creencias y valores culturales sobre la organización). 


\section{LOS PROCESOS DE CONTROL}

Descritos los cuatro tipos de modelos analíticos de las organizaciones, que responden a los procesos sociales básicos y a las principales formas de racionalidad, es posible abordar el proceso fundamental que rige las organizaciones occidentales, tanto en los países socialistas como en los capitalistas. Se trata del proceso de control.

Es preciso recordar, en primer lugar, que la teoría weberiana de las organizaciones y de la sociedad es, sin duda, una teoría de la dominación y el control, a pesar de que la corriente predominante entre sus seguidores ha pretendido reducirla a una teoría del consenso y del liderazgo. Sus aportaciones constituyen una ampliación de la perspectiva marxista centrada en los conflictos entre propietarios y trabajadores.

Weber abre el horizonte analítico a todas las sociedades en las que pueden apreciarse modelos institucionalizados de Zweckrationalität. Además de las relaciones de clase, estudia las relaciones conflictivas más comunes, derivadas de las posiciones jerárquicas dentro de las organizaciones.

Hickson y McCullough resumen así el puesto central que Weber atribuye al poder y al control en las organizaciones y en la sociedad: "El poder es la esencia de la organización, desde el análisis que Weber hace de su legitimación básica en términos carismáticos, tradicionales o burocráticos, hasta el control mutuo de una persona por otra inherente en la interacción social de cada día en el trabajo» (Hickson y McCullough, en Salaman y Thompson, 1980: 27-28).

La perspectiva teórica adoptada integra la teoría de la acción social y el análisis estructural de las organizaciones (Giddens, 1979; Hernes, 1976), tratando de explicar en qué forma las variables macrosociales afectan a los comportamientos individuales y en qué medida éstos, a su vez, inciden sobre las estructuras.

G. Hernes señala, como caso prototípico de interacción entre los dos niveles, la transición demográfica. El descenso de la tasa de fecundidad implica una serie de decisiones de las diferentes unidades familiares, esto es, decisiones microsociales. La agregación de todas estas decisiones individuales es la que produce un efecto macrosocial, al tiempo que este condicionante estructural facilita tales decisiones individuales.

La integración de estos dos tipos de procesos constituye la perspectiva metodológica de W eber. En una línea semejante, estas páginas intentan poner de manifiesto las interrelaciones entre los procesos instrumentales y expresivos, mediante los cuales se llevan a cabo la dominación y el control en las organizaciones de carácter racional-legal.

Las estrategias técnicas han evolucionado, desde el diseño de modelos racionales de control directo hasta formas más penetrantes y menos perceptibles. 
Dada la creciente importancia de estas últimas, se prestará especial atención a los modelos de la economía política y de las anarquías organizadas.

Hasta hace relativamente poco, los modelos racionales, asimilados en gran medida al tipo ideal de burocracia de Weber, han sido claramente predominantes. Su presupuesto latente, como señalan Thompson (1967) y Aldrich (1979), era la existencia de objetivos no ambiguos, a los que respondían directamente tanto la estructura organizativa como las decisiones. Su racionalidad era de carácter económico: lograr el óptimo de eficiencia. Tales modelos buscaban más el control de las organizaciones que su comprensión. Se trata de modelos cerrados que toman el entorno como un dato.

A un tipo similar de racionalidad responden también - como ya se ha indicado- los modelos de la integración social, cuya forma específica de control desarrolló una teoría psicosocial del liderazgo. La autoridad del líder y su capacidad de integración del grupo no derivan de una posición jerárquica, sino del reconocimiento de sus cualidades personales.

$\mathrm{El}$ descubrimiento de «que los miembros de las organizaciones tienen necesidades, motivaciones y tendencias, y que están limitados en sus conocimientos y en su capacidad para comprender y resolver los problemas» (1969: 134) llevó a March y Simon a establecer el principio de una racionalidad limitada. Su fundamento está en el hecho de que «el comportamiento racional se apoya sobre esquemas simplificados que toman en consideración los principales rasgos de un problema sin dar cuenta de su complejidad» (1969: 165).

La elección de una acción y sus consecuencias se descubren de manera secuencial, a través de procesos de búsqueda. El orden o la secuencia seguida delimitan sustancialmente la solución escogida, dado que el hallazgo de una alternativa satisfactoria interrumpe la búsqueda.

En este punto cobran una importancia decisiva la información y sus canales de transmisión (165-168), porque la información no es dada, sino que es preciso obtenerla. Como escriben Katz y Kahn (1966: 223): «Cuanto más nos acercamos al centro de control y de toma de decisiones de una organización, más se acusa la importancia del intercambio de información». En definitiva, los inputs de cada secuencia dependen en gran medida del formato de la red de comunicaciones.

En el proceso de búsqueda el tiempo constituye, asimismo, un elemento importante. Alcanzar un objetivo ahora supone pasar por alto otros, tanto ahora como en el futuro.

La propia noción de racionalidad limitada plantea la cuestión del control. Importa destacar a este propósito que la estructura de las organizaciones ayuda a simplificar las decisiones de los miembros, lo que implica una doble dimensión de eficiencia técnica y de control político, en las que se originan — según Gouldner- dos concepciones distintas de la burocracia.

Scott (1981), desde una óptica consensual, adopta la primera hipótesis, en un sentido similar al que March y Simon (1969: 139-147) atribuyen a las 
nociones de esquema y estrategia de ejecución. A pesar de su orientación marxista, Konrad y Szelenyi comparten de hecho esta misma hipótesis, al estimar que el poder creciente de los intelectuales responde a su capacidad de apoyar sus decisiones en datos «objetivos».

Perrow (1979), por el contrario, inspirándose a un tiempo en Marx y Max Weber, interpreta los procesos de simplificación como formas estructurales de control de las decisiones de los miembros. Entre los mecanismos de control señala la existencia de presupuestos valorativos y fácticos, que condicionan la toma de decisión. Los primeros responden a los objetivos deseables y definen la Wertrationalität de la organización. Los presupuestos fácticos se refieren a la lógica que rige la relación entre los medios y los fines (Zweckrationalität).

El grado de acuerdo o desacuerdo en los presupuestos conduce a una situación de consenso o conflicto sobre las decisiones. En los presupuestos valorativos o, lo que es lo mismo, en la elección de los fines cabe un margen mayor de libertad. Pueden escogerse mediante:

1) Un fiat autocrático, que tiende a producir conflicto.

2) Un proceso democrático que conduce a un mayor consenso, si bien, como señalan Selznick y Gouldner, suelen también a más largo plazo dar origen a disfunciones latentes.

3) La formación de coaliciones que negocian en una situación de equilibrio inestable entre el consenso y el conflicto.

Los presupuestos de carácter fáctico, al referirse a la selección de los medios más eficaces para el logro de los objetivos, implican un mayor componente técnico y están sometidos a la prueba de la verificación empírica. En consecuencia, se da en ellos un grado mayor de consenso.

En la práctica, sin embargo, no es fácil establecer una diferencia neta entre medios y fines porque entre ellos se establece una jerarquía continua, a lo largo de las diversas unidades organizativas. Lo que para un nivel jerárquico determinado define un objetivo, es percibido sólo como un medio por los niveles superiores. De este modo, la jerarquía de los fines acaba coincidiendo con la de los medios.

De todas formas, constituye un imperativo que los miembros de los niveles más bajos no pongan en cuestión el objetivo último de la organización. La forma de eliminar este riesgo es controlar las bases en que se apoyan las decisiones de los miembros. Aquí radica el carácter esencial de la noción de control y de los diversos mecanismos organizativos de simplificación $\longrightarrow$ de control estructural- de las decisiones.

Los estudios sociológicos más recientes sobre el liderazgo siguen esta orientación. Inspirándose en el desarrollo de las organizaciones burocráticas señala- 
do por Weber, derivan el poder del líder de su posición jerárquica y del papel que desempeña, en lugar de atribuirlo a su valía personal. La progresiva racionalización de su función asimila su actividad a la de un experto cuya influencia está fuertemente asociada a la centralidad de su posición en la red de comunicaciones.

Según D. Bell y A. Tannenbaum, entre otros, la bibliografía sobre el liderazgo muestra un claro sesgo elitista y conservador, que responde a la estructura autoritaria de los modelos clásicos de organización. Tal es el sentido del postulado de Michels sobre la incompatibilidad entre liderazgo y democracia.

Quizás por esta implicación no pocos de los más recientes y significativos trabajos en este campo se han orientado hacia el análisis de los procesos de control organizativo. Tannenbaum y Cooke, a partir de una larga serie de estudios comparados cross-national, llegan en 1979 a la conclusión de que la eficiencia de una organización depende de su grado de control, sin que las teorías inspiradas en los tipos de liderazgo democrático-autocrático permitan realizar previsiones en este sentido.

El concepto de control organizativo permite establecer un puente entre la perspectiva de la acción social y el análisis estructural. Las estructuras organizativas son en realidad mecanismos formalizados de control, idea a la que responde el tipo ideal de burocracia.

March y Simon adoptan ya en gran medida esta perspectiva, si bien sus análisis, por el acento en las motivaciones, han sido clasificados como psicosociales. Algunos de sus conceptos, en particular el ya mencionado de la absorción de incertidumbre, se refieren al control de los presupuestos de la decisión. En una línea similar actúan el diseño de los flujos y de los contenidos informativos, la definición de expectativas y la limitación en la búsqueda de alternativas.

\section{TIPOS DE CONTROL}

Resumiendo lo expuesto, pueden señalarse tres tipos básicos de control:

1. Control directo: Se ejerce mediante la imposición de órdenes y la vigilancia expresa.

2. Control estructural: Corresponde a los niveles más formalizados de la organización. Es ya en buena medida discreto (unobtrusive).

3. Control completamente difuso (fully unobtrusive): No opera directamente sobre el proceso concreto de la toma de decisión, sino sobre sus presupuestos culturales. Es un tipo de control más difícil de conseguir, pero mucho más eficaz. 
Como prototipo del control directo o completamente abierto pueden mencionarse los sistemas de vigilancia. El Panóptico de Bentham, diseñado originariamente para las prisiones, aunque el propio autor intuyó su posible adaptación a otras instituciones, es tal vez el ejemplo más acabạdo de este tipo de control. Puede aplicarse -escribe Bentham- «a todos los casos en que un gran número de hombres debe estar constantemente bajo la inspección de unos pocos, sea para el castigo de los culpados, sea para reformar a los malos, sea para forzar a los perezosos al trabajo, sea para facilitar la asistencia de los enfermos o sea para hacer fácil la enseñanza y llevar el poder de la educación a un punto inconcebible hasta el día» (1979: 80).

La pretensión de Bentham de aplicar este modelo a todos los ámbitos de la vida social anticipa uno de los dilemas más dramáticos de las sociedades avanzadas. Se trata de la contradicción entre el ideal democrático y la búsqueda -mediante el uso generalizado del control informático- de la transparencia permanente de las acciones de los individuos frente al ojo del poder.

Sus ideas entraron en España en la época liberal. Según sus principios se construyeron diversas cárceles, entre ellas la Modelo de Barcelona. Hoy, sin embargo, sobre todo en las sociedades democráticas, los mecanismos organizativos de control no pueden ser tan diáfanos.

«El control organizativo —escribe Thompson (1980: 220) - no necesita ejercerse expresamente en cada situación y decisión; se encuentra preestablecido en las premisas de la toma de decisión.» «Tales premisas —añade Perrow (1972: 175-176) — vienen definidas por el "vocabulario" de la organización, las reglas, los criterios de selección de personal -en resumen, por los aspectos estructurales.»

Tal vez la estructura de control organizativo más claro sea la división del trabajo, por la interdependencia que genera entre las diferentes unidades. Por esta razón constituyó el centro de atención de la Escuela de la Organización Científica del Trabajo. El recorrido y la densidad de las redes de comunicación obedecen a la misma lógica.

Richard Edwards, en su conocida obra Contested Terrain (1979), señala dos tipos básicos de control estructural:

a) Técnico que afecta sobre todo a los blue collars y se apoya en la tecnología del proceso productivo.

b) Burocrático, dirigido a los white collars, esto es, a las funciones administrativas.

De acuerdo con la inspiración marxiana de este autor, cada forma de control responde al desarrollo de un determinado tipo de empresas. El control directo se ajusta al predominio de la pequeña empresa; los controles estructurales son propios del gran capitalismo.

El tercer tipo señalado de control se basa en los procesos culturales que definen los presupuestos cognitivos de las organizaciones. Su estudio ha dado 
origen a uno de los debates centrales de nuestro tiempo: la reducción de la racionalidad técnica, la absorción del sentido por la estructura.

Si bien la discusión nació y se desarrolló en los campos de la filosofía social y de la ciencia, hoy constituye también un objeto prioritario de análisis de la sociología de las organizaciones. "La estructura no será más que una forma vacía -advierte John Child- si no concuerda con las expectativas derivadas de la propia cultura» (en J. Child y A. Kieser, 1979: 253).

A. M. Pettigrew (1979) aplica al estudio de las organizaciones la noción de cultura, haciéndola analíticamente operativa mediante su descomposición en una familia de conceptos: símbolo, lenguaje, creencia, ideología, ritual y mito.

En la cultura organizativa se fundamentan los procesos de compromiso e identidad con la organización. Desde una perspectiva procesual, la cultura no sólo necesita ser creada, sino mantenida y renovada; de lo contrario se disuelve. Las palabras y la cultura, por vincular las actitudes a la acción, son parte de la acción. El compromiso con la organización aparece así claramente dependiente de la creación de una cultura y de la capacidad de los líderes para usar el poder de las palabras.

Uno de los indicadores más significativos de la existencia de una cultura organizativa es el desarrollo de un vocabulario propio, que expresa y, al mismo tiempo, consolida el sentido de exclusividad en los miembros.

En las organizaciones normativas, sobre todo en las religiosas y políticas, la importancia del lenguaje es clave, porque en él la doctrina oficial cristaliza en palabras que adquieren un valor dogmático. El credo doctrinal, diseñado con la intención manifiesta de expresar los valores comunes, acaba convirtiéndose en el mecanismo de control más claro y eficaz de la ortodoxia o de la desviación.

En un sentido semejante y cercano al de la cultura organizativa pueden interpretarse los términos acuñados Selznick y Clark, respectivamente, de carácter de la organización y saga. Dichos conceptos representan la encarnación de los valores comunes o de la comprensión colectiva de los miembros.

Una nueva organización equivale al paso de una situación sin creencias ni reglas establecidas a otra en la que aparecen nuevos valores y nuevas creencias. Si para Cassirer lo específico del ser humano es su capacidad de inventar y comunicar determinantes de su propia cultura, esto es, valores, el hombre - para Pettigrew - es un creador y manager del significado.

Dentro de la cultura organizativa, los rituales desempeñan un importante papel de cohesión en cuanto celebraciones compartidas de la pertenencia común. Son la expresión más acabada $-\mathrm{y}$ en buena medida sacralizada- del compromiso de los miembros con la organización, similar al proceso descrito por Durlcheim en las Formas Elementales de la Vida Religiosa.

La cultura, además de afectar en profundidad a los procesos intraorgani- 
zativos, resulta de capital importancia para la comprensión de los procesos de interacción de las organizaciones con su entorno.

\subsection{El isomorfismo organizativo}

Meyer y Rowan $(1977,1981)$ observan que no pocas estructuras organizativas formales surgen como reflejos de valores y normas institucionales o culturalmente establecidos, dando origen a un proceso de isoformismo organizativo. Se diría que la presión cultural del entorno obliga a las organizaciones a volverse isoformas respecto de las instituciones sociales.

B. J. DiMaggio y W. W. Powell resumen así este fenómeno: «tan pronto como un set de organizaciones surge en un campo institucional, sucede algo paradójico: los actores racionales, al tratar de cambiar sus organizaciones, las hacen cada vez más semejantes» (1983: 147).

La lógica de este proceso responde a la búsqueda de mayores perspectivas de supervivencia mediante un incremento de legitimidad. Tal como prescribe el funcionalismo normativo, la legitimidad deriva de la asimilación al sistema central de valores.

Este fenómeno podría interpretarse como una forma de racionalidad a largo plazo, frente al simple ajuste más o menos coyuntural de corto alcance.

Muchas de las proposiciones, programas y procedimientos de las organizaciones modernas responden a creencias y valores públicos. Son, en definitiva, manifestaciones de poderosas reglas sociales institucionalizadas, que funcionan como mitos altamente racionalizados.

Tales mitos tienen dos propiedades fundamentales. En primer lugar, identifican ciertas metas operativas como las formas más apropiadas de realizar determinados valores.

En segundo lugar, aparecen altamente institucionalizadas y, en consecuencia, más allá del arbitrio de cualquier miembro individual. En definitiva, se dan por supuestas, lo que les concede un elevado grado de legitimidad, justamente por aparecer más allá de toda discusión.

Un ejemplo típico de esta situación podría ser la transformación de la prostitución, de profesión prerracional en una profesión altamente racionalizada mediante el control médico y la organización burocrática, cambiando, al mismo tiempo, su denominación en clínicas de terapia sexual, salones de masaje y similares.

La asimilación institucional da origen a un lenguaje organizativo, cuya evolución es tal vez la expresión más visible del proceso de isomorfismo. El vocabulario oficial ofrece reglas de legitimación y de racionalidad aceptables. Las metas proclamadas por líderes y coaliciones que emplean un vocabulario aceptado se suponen orientadas hacia fines colectivos. Por el contra- 
rio, las que omiten los términos consagrados por el entorno corren el riesgo de ser percibidas como desviadas y, en consecuencia, criticables.

Para Dowling y Pfeffer (1975) y en general la teoría de la dependencia de los recursos, las grandes organizaciones luchan por la definición de los mitos institucionales. En la medida en que de esta manera sean capaces de imponer valores que responden a sus productos o a sus intereses conseguirán una posición más ventajosa.

Una falta de coherencia o, más exactamente, un ajuste flexible entre las diferentes estructuras organizativas puede, así, interpretarse como una forma de adaptación racional e incluso de control discreto del medio.

Tres proposiciones resumen la forma en que se desarrolla el proceso de isomorfismo estructural.

1. Cuanto más se moderniza una sociedad, más se extiende la red institucional en cuanto forma altamente racionalizada.

$2 .^{a} \quad$ A medida que aumentan las reglas institucionales o los mitos racionales, las organizaciones formales tienden a incorporar en su estructura tales reglas.

3. ${ }^{a}$ Las organizaciones que incorporan elementos racionales socialmente legitimados en sus estructuras formales maximizan su legitimidad e incrementan sus fuentes de supervivencia.

\subsection{Supervivencia y contormidad ceremonial}

Es preciso tener presente que cuanto más crecen las organizaciones, más crucial resulta para ellas la cuestión de la supervivencia, la cual llega a convertirse en su objetivo predominante.

Según el tipo de organización, será más importante la respuesta a las demandas internas y a las relaciones inmediatas con el entorno, o, por el contrario, una conformidad ceremonial con las demandas institucionales. En el primer caso, el output puede ser fácilmente evaluado en términos técnicos y tiende a desarrollar un mercado. La eficiencia determina el éxito.

En el segundo caso, por ejemplo, las organizaciones escolares o las agencias burocráticas del Gobierno, suelen utilizar tecnologías ambiguas para producir outputs difíciles de valorar. En una línea similar, las incertidumbres de tecnologías cambiantes e impredicibles suelen generar demandas de institucionalización para evitar el riesgo.

Entre estos dos tipos de organizaciones puede establecerse un continuo que va desde las caracterizadas por el criterio de la eficiencia hasta las más marcadas por una adaptación isofórmica a las reglas institucionales. Es importante señalar que, incluso las que se fundamentan en un carácter más técnico, dependen en buena medida también del entorno. La definición social de la rea- 
lidad afecta incluso a las propiedades de carácter técnico y de la propia eficiencia.

El ejemplo más claro es el de la conformidad normativa que imponen los diferentes Colegios Profesionales, en un doble intento de aumentar la legitimación o el prestigio de sus miembros y restringir la competencia.

Esta contaminación cultural de las estructuras organizativas responde claramente a la perspectiva weberiana, que sitúa en el centro del análisis sociológico la cuestión de la legitimidad y la influencia de los valores. Para Max Weber, legitimidad y dominación (o control en términos organizativos) constituyen las dos caras de una misma realidad: las dos necesidades fundamentales de cualquier organización.

El modelo estructural de análisis de las organizaciones olvidó la primera dimensión. La racionalidad formal, que trata de ajustar los medios a los fines, no agota todo el ámbito de la racionalidad. La acción del actor social implica también un significado en el que se enraíza la cuestión de la legitimidad. Hablar de instituciones significa plantear la cuestión de los valores a los que responden. «Las organizaciones -escribe Van Doorn- son siempre parte del mundo de la acción social conscientemente estructurada» (1979: 62).

Recientemente numerosos estudios siguen esta perspectiva cultural que supone una vuelta al horizonte weberiano. Así, Crocier descubre en las organizaciones francesas especiales rigideces derivadas de los efectos culturales del entorno. Peter Clark, en un estudio comparado entre los monopolios de tabaco en Francia e Inglaterra, ratifica el valor de la explicación cultural de Crocier, que da cuenta de importantes diferencias observadas en ambos países. R. A. Schoenherr y J. Pérez Vilariño (1979) descubren diferencias significativas en el grado de compromiso con organizaciones religiosas en España y EE UU, que responden a las variaciones del entorno. M. Maurice insiste en la importancia del «efecto societal» frente a la tesis de la free culture, inspirada en la creciente generalización del tipo ideal de burocracia, como resultado de la universalización de la tecnología.

En una línea similar, J. C. Castillo hace especial hincapié en el «condicionante cultural» y «la politización progresiva de la empresa». A la luz del primero de estos dos fenómenos, y a partir de los estudios de Linz y De Miguel, observa la existencia en España de «dos modos distintos de entender la gestión de la empresa, uno tradicional... [y] otro moderno». Por su parte, «la sustitución progresiva de la lógica de la utilidad por la lógica del poder» responde, en su opinión, a la creciente atención a los procesos políticos (Castillo, 1976). 


\section{EL MODELO DE LA ECONOMIA POLITICA}

Expuestos los tres tipos básicos de control, se presentan a continuación los dos modelos fundamentales de racionalidad organizativa, que responden más directamente a los tipos de control de mayor vigencia hoy: los estructurales y los culturales o completamente discretos (fully unobtrusive). Estos modelos son: la Economía Política de las Organizaciones y las Anarquías Organizadas.

Si el problema de los modelos racionales de la teoría clásica es buscar un ajuste óptimo, o al menos satisfactorio, de los medios a unos fines dados, el problema fundamental de la perspectiva política es la determinación de los propios objetivos. El recurso básico para ello es el ejercicio del poder, por lo que, según Karpik, las organizaciones son sistemas políticos.

El modelo de las «contingencias estratégicas», desarrollado por Hickson y otros (1971 y 1980), es el antecedente más claro de la perspectiva de la Economía Política. «Los procesos de decisión estratégica —escriben Hickson y McCullough- son en el límite procesos de poder político. En ellos se presta más atención a un individuo que a otro y los miembros de unidades poderosas consiguen hacer valer sus propuestas... La decisión óptima, realizada a partir de un cálculo exacto de toda la información significativa, sin dejarse contaminar por otro tipo de factores, probablemente no existe ni nunca existió fuera de los libros de matemáticas» (Hickson y McCullough, en Salaman y Thompson, 1980: 50 y 52).

La organización se concibe como un juego de ajedrez. El poder de cada pieza viene definido por su posición, en lugar de conseguir su posición en virtud de su poder. Las reglas del juego atribuyen posiciones especiales - grados distintos de poder- a las diferentes piezas. En las organizaciones, y en general en la sociedad, quien ocupa un puesto con mayor capacidad de control sobre la incertidumbre tendrá mayor poder para modificar las reglas en su favor.

Desde esta visión del poder estratégico de las diferentes subunidades no resulta difícil el salto a la idea de coalición. La necesidad de coaliciones para la toma de decisiones importantes deriva de la complejidad de las organizaciones. Ningún ejecutivo cuenta con poder suficiente para forzar las decisiones en solitario. En consecuencia, la toma de decisiones se convierte en un proceso político, regido por la negociación y el compromiso.

Por su parte, los conflictos de intereses adquieren carta de ciudadanía dentro de la organización, en la que coexisten numerosas metas opuestas, frecuentemente encarnadas en departamentos diferentes. Esta concepción dista mucho de las teorías clásicas (sistematizadas por March y Simon), que asumen la existencia de objetivos claros y metas jerarquizadas.

Con todo, para Cyert y March -iniciadores del modelo de coalicionesla importancia de los conflictos en el ámbito de los objetivos se reduce por 
la separación o relativo aislamiento de los puntos de vista opuestos y por su tratamiento secuencial. Los enfrentamientos se resuelven en una crisis de gestión, con un remodelamiento de la coalición dominante. Al no buscarse la solución más eficiente, sino la más satisfactoria políticamente, los conflictos no alcanzan nunca una solución definitiva.

El entorno - como fuente externa de recursos y de incertidumbre- cobra así una especial importancia para las grandes organizaciones.

Zald ve precisamente en la interacción entre la economía política interna y externa la clase explicativa del cambio de objetivos de una organización como la YMCA.

La necesidad de una política externa surge, en primer lugar, de la red de compradores y suministradores, o de clientes en general. Al hacerse ésta demasiado compleja obliga a establecer coaliciones, vinculando a ellas importantes áreas internas de la organización. El segundo polo de la política externa viene definido por el Estado, el cual establece diversos grados de regulación para los distintos tipos de organizaciones.

Para Zald, la importancia de los modelos de la Economía Política deriva de la atención que prestan a los dos procesos más importantes: los económicos y los políticos.

A. Pettigrew estudia el desarrollo de las coaliciones, esto es, cómo cualquier incidente observable se inserta en una larga cadena de interacciones. Según este autor inglés, el modelo político puede resumirse de la siguiente manera.

Los diferentes departamentos, derivados de la división del trabajo, desarrollan intereses específicos que responden a sus funciones y responsabilidades particulares. El éxito de la demanda de recursos organizativos que plantea cada uno de ellos depende de su habilidad para conseguir el apoyo de los demás, o de formar una coalición fuerte. Para ello no basta con ganar aliados y establecer pactos. Es más importante dominar la ideología de la organización, haciendo creer a los demás que la contribución del propio departamento es esencial. (Es aquí donde cobra todo su sentido la atención de Pettigrew a la cultura organizativa.)

El análisis histórico y macrosocial le permite a Lucien Karpik vincular las reglas y prácticas organizativas a la evolución del entorno. La modificación reciente más significativa de éste es el paso del capitalismo mercantil e industrial al tecnológico con el predominio de las organizaciones multinacionales, las cuales dan origen a una cultura diferente. Al unir investigación científica, economía y política no se limitan a responder y legitimar la demanda. Producen una cultura de la dominación que busca controlar los valores de los consumidores.

En el interior de las organizaciónes, la noción clave es la de lógicas de acción —o racionalidades observables desde fuera-, que se diferencian de la percepción que el sujeto tiene de sus motivaciones personales. La organización, 
desde estas lógicas, es un sistema ajustado flexiblemente en el que los individuos forman coaliciones para defender sus intereses.

A pesar del enfoque claramente conflictivo de este modelo, la delimitación de los márgenes dentro de los que se mueven los conflictos deja traslucir un horizonte funcionalista. Si la historia de una organización incide en sus luchas actuales, también la obligación que sienten las diferentes partes de mantenerse unidas en el futuro impone ciertos límites al nivel de conflictos. Por fuertes que sean los conflictos, todos los miembros, incluso aquellos que se consideran perdedores, no olvidan la situación ulterior al conflicto, esto es, el interés por mantener la organización. En definitiva, los conflictos cumplen la función - señalada por Coser- de adaptación del sistema a los cambios en el entorno.

El presupuesto fundamental de los modelos de la Economía Política asume que las organizaciones son incapaces de producir todos los recursos y desempeñar todas las funciones que necesitan. Por ello se ven obligadas a establecer relaciones y concertar transacciones con otros subsistemas del entorno. Además de las interdependencias derivadas de la división del trabajo, este modelo postula que ciertas interpedendencias son buscadas o evitadas en virtud del poder y control que tal situación ofrece.

El modelo ecológico de la selección natural, o modelo orgánico, establece una selección ambiental — determinista- de las características más evolucionadas o ajustadas al medio. El mayor problema que plantea es la dificultad de evitar el argumento tautológico: si las estructuras que no se adaptan perecen, las que sobreviven son por definición adaptadas.

Por el contrario, el modelo de la dependencia de los recursos -el que más se adapta a la perspectiva de la Economía Política - presupone la existencia de una relativa abundancia o escasez de recursos, entre los que la lucha por la supervivencia obliga a la organización a escoger, mediante una toma de decisiones estratégicas, según la terminología de Hickson (1971) y Child (1972).

La diferencia más significativa entre ambas perspectivas reside en el margen de maniobra (slack) de que dispone la organización frente al entorno, a cambio de una racionalidad más limitada que se contenta con una decisión política o estratégica.

La conexión entre el modelo de la Economía Política y la teoría de la dependencia de los recursos es casi obvia: sólo es posible subrayar la importancia de la toma de decisiones si existen varias alternativas viables. $\mathrm{La}$ incertidumbre que éstas generan es la que hace importantes, tanto los procesos políticos internos como los procesos políticos con el entorno.

El carácter activo de la organización frente al entorno puede llevar no sólo a tratar de conseguir un ajuste, sino incluso a dominarlo y modificarlo. Tal es la estrategia que siguen las grandes organizaciones multinacionales en relación con la política de los diferentes Estados, que pretenden acotarles entor- 
nos demasiado restringidos a sus planteamientos supranacionales. Una de las vías más eficaces de control del entorno es justamente la imposición de una definición determinada de éste. Aquí radica la creciente importancia que las grandes organizaciones otorgan a la creación de una cultura propia.

\section{AJUSTE FLEXIBLE Y ANARQUIAS ORGANIZADAS}

El desarrollo de las formas de dominación simbólica orienta el análisis, sobre todo de las organizaciones normativas -Universidades y sistema educativo en general, asociaciones voluntarias, etc.-, hacia una forma de racionalidad más difusa y aparentemente anárquica, en contraposición a los cánones estrictamente racionales del modelo económico. A este tipo responden los modelos de ajuste flexible -loosley coupled - descritos por March y Olsen (1975), Weick (1976), Meyer y Rowan (1977), DiMaggio y Powell (1983).

La mayoría de los estudios sobre organizaciones insisten en la interdependencia funcional y, en general, en los aspectos racionales de las organizaciones, que perviven a pesar de una deficiente conexión entre sus unidades.

Con el concepto de ajuste flexible introducido por Glassman (1973), se hace referencia a dos acontecimientos que interactúan entre sí, pero conservando su identidad propia y alguna forma de separación física o lógica.

A partir de este concepto, Cohen, March y Olsen hablan de «anarquías organizadas», refiriéndose a organizaciones cuyas estructuras están diseñadas con una elevada elasticidad para el cambio. Se caracterizan por tener unos objetivos o preferencias problemáticos, límites imprecisos y una tecnología poco definida. Las preferencias de los miembros varían fuertemente entre sí, lo mismo que su dedicación a la organización. Por su parte, tampoco resultan unívocos los principios tecnológicos, tales como la definición de una buena técnica docente o la eficiencia de un equipo investigador.

Los modelos basados en la toma racional de decisión o en la negociación política resultan de difícil aplicación a estas anarquías organizadas. El concepto de ajuste flexible desempeña — tal como señala Weick (1976) — una serie importante de funciones, cuyo común denominador es la facilidad para introducir innovaciones en unidades parciales, aislando la tensión a que pudieran dar origen.

Combinado con un sistema jerárquico da lugar a los sistemas que Simon (1962) denomina desmontables. Tales modelos son los que predominan en la naturaleza entre los sistemas más complejos, porque resultan especialmente aptos para integrar las innovaciones. Permiten también comprender uno de los fenómenos más significativos de las complejas sociedades postindustriales: la creciente densidad e interconexión de las redes sociales, flexiblemente ajustadas.

A estas «anarquías organizadas», o sistemas flexibles de retención de mu- 
taciones contrapuestas - cuyo prototipo son las Universidades-, es al que los autores señalados designan con el pintoresco nombre de «modelo del cubo de la basura» ( $A$ Garbage Can Model).

«Para entender los procesos al interior de las organizaciones - escriben Cohen, March y Olsen-- es dado imaginar una oportunidad de elección como un cubo de la basura, al que los miembros tiran diversas clases de problemas y soluciones, a medida que se van produciendo. La mezcla de la basura en cada cubo depende de la mezcla de basura disponible, de las etiquetas puestas a los diferentes cubos, del tipo de basura que se produce habitualmente y de la rapidez con la que se recoge la basura y se retira de la escena» (Cohen y otros, 1972: 2).

Las ideas centrales de este modelo pueden sistematizarse de la siguiente manera:

a) Los problemas son percibidos como receptáculos capaces de recibir las soluciones por las que se interesan los diferentes miembros.

b) En consecuencia, el cubo lleno de problemas se convierte en una oportunidad $o$ en un recurso disponible.

c) La atención de los miembros a un cubo o a otro depende del número de cubos existentes, de las mezclas de problemas que contienen y del tiempo disponible.

d) A lo largo de este proceso, los problemas se despersonalizan alcanzando vida propia. La pluralidad de respuestas que los nuevos miembros pueden ir dando a viejos problemas sorprendería a quienes definieron por primera vez tales problemas.

e) Por último, numerosos problemas, y más todavía las soluciones concretas, se van eliminando sistemáticamente.

Con este modelo, las decisiones son el resultado de la confluencia de cuatro procesos relativamente independientes:

a) El flujo de problemas que pueden provenir de dentro o de fuera.

b) El flujo de soluciones de que dispone la organización.

c) El flujo de miembros que vienen y se van.

d) El flujo de oportunidades de elección, derivadas de las demandas que experimenta la organización.

Los autores presentan un programa de ordenador para la simulación de este modelo, y en particular de las diferentes formas de vincular estos flujos entre sí. Tal vez uno de los hallazgos más significativos del experimento sea la aparición de problemas y soluciones desconectados. 


\section{DISCUSION}

A la vista de los diferentes modelos de racionalidad y de las diversas formas de control organizativo, surgen unas preguntas fundamentales: ¿a qué situaciones se ajusta más cada uno de los modelos y en qué contextos es más verosímil la aparición, por ejemplo, de una anarquía que de un modelo racional o político, así como el desarrollo de un tipo de control más o menos directo o discreto?

Como hipótesis de trabajo para estudios empíricos podrían formularse las tres proposiciones siguientes:

1. Cuanto mayores y más generalizados sean los problemas (esto es, cuanto más graves sean sus consecuencias), más fuerte será la presión experimentada por el sistema para recurrir a mecanismos de control.

2. ${ }^{a}$ Cuanto más abierta sea la amenaza contra la seguridad de la coalición dominante, mayor será también el empleo de todo tipo de mecanismos de control.

3. ${ }^{\mathrm{a}}$ Por último, cuanto más difíciles sean las consecuencias a corto plazo, mayor será la tolerancia con las anarquías organizadas de los «cubos de basura».

Es preciso advertir que las diferentes organizaciones (incluidas, desde luego, las Universidades) muestran en sus procesos de toma de decisión variaciones históricas en sus grados de racionalidad, negociación política y anarquía. Tales procesos estarán, a su vez, afectados por niveles y formas diferentes de control, según la importancia y el tipo de problemas a resolver.

La investigación se orienta cada vez más hacia las formas insidiosas de control completamente discreto, vinculadas a los presupuestos cognitivos de la interacción. La distancia que media entre los elementales modelos de vigilancia o control directo y las nuevas formas de racionalidad simbólica - que intentan dar alcance a las bases cognitivas de la acción humana- resume la reciente evolución de los modelos organizativos.

Mientras el análisis sociológico no penetre en el discreto o latente ámbito de los mecanismos simbólicos de control, se le podrá aplicar la parábola del zoo que presenta Perrow (1980): «Seremos como ciegos que manosean el elefante, que denominamos "organización", y llenos del sentido del deber informamos sobre sus verrugas, su trompa, sus rodillas y su cola, convencido cada uno de nosotros de haber encontrado la naturaleza exacta de la bestia. Pero lo peor es que ni siquiera estamos mirando a la misma bestia.»

Es preciso recordar - continúa Perrow - que la «teoría de las organizaciones - si quiere situarse en la tradición de Weber, Blau o Gouldner- no es un deporte de contactos sociales», como pretende la Escuela de las Relaciones Humanas y parecen aceptar los centros de formación empresarial. Tal 
como se indica al comienzo de estas páginas, la esencia de la organización, según Weber, es el poder, el cual parece cada vez más convencido de que su eficacia no depende de su grado de visibilidad.

\section{BIBLIOGRAFIA}

Aldrich, H. E. (1979): Organizations and Environments, Prentice Hall, New Jersey.

Bent ham, J. (1979): El Panóptico, La Piqueta, Madrid.

Castillo, J. C. (1976): Sociología de la Empresa, Unidades Didácticas de la UNED, vol. II.

Coh EN, M., y otros (1972): «A Garbage Can Model of Organizational Choice», Administrative Science Quarterly, 17 (marzo): 1-25.

Coleman, J. (1983): The Asymmetric Society, Siracuse University Press, Nueva York.

Cyert, R. M., y March, J. G. (1963): A Behavioral Theory of the Firm, Prentice Hall, Englewood Cliffs.

CHILD, J. (1972): «Organization structure, environment, and perfomance: the role of strategic choice», Sociology, 6 (enero): 1-22.

CHILD, J., y Kieser, A. (1979): "Organizational and Managerial Roles in British and West German Companies: an examination of the culture --free thesis», en C. Lammers y D. Hickson: 251-271.

Del Campo, S. (1968): Cambios Sociales y Formas de Vida (Estudios de Sociología), Ariel, Barcelona.

DiMaggio, B. J., y Powell, W. W. (1983): «The iron cage revisited: Institutional isomorphism and collective rationality in organizational fields», en $A S R$, vol. 48, núm. 2: 147-160.

Dowling, J., y Pfeffer, J. (1975): «Organizational legitimacy: Social values and Organizational Behavior», Pacific Sociological Review, 18: 122-136.

EDWARDS, R. (1979): Contested Terrain: The transformation of the Workplace in the Twentietb Century, Basic Books, Nueva York.

Giddens, A. (1969): Control Problems in Social Theory, University of California Press, California.

Glassman, R. (1973): «Persistence and Loose Coupling», en Bebavioral Science, 18: 83-98.

Hall, R. H. (1972): Organizations. Structure and Process, Prentice Hall, New Jersey.

Hernes, G. (1976): «Structural change in social processes», en American Journal of Sociology, 82 (noviembre): 513-547.

Hickson, D. J., y otros (1971): «A strategic contingencies theory of intraorganizational power», en Administrative Science Quarterly, 16: 216-229.

Hickson, D. J., y McCulloug y K. Thompson: 27-55.

Hrrschman, A. (1970): Exit, Voice and Loyalty, Harvard University Press, Cambridge (Mas.).

Hobsbawn, E. J. (1975): The Age of Capital, 1848-1875, Weidenfeld and Nicolson, Londres.

KARPIK, L. (1978): Organizations and Environment. Theory, Issues and Reality, Sage Publications Inc., California.

Katz, D., y KaHn, R. (1966): The Social Psycbology of Organizations, Wiley, Nueva York.

LAmmers, C., y Hickson, D. (1979): Organizations Alike and Unilke: International and Inter-Institutional Studies in the Sociology of Organizations, Routledge \& Kegan Paul, Londres.

March, J., y Olsen, J. (1975): Choice Situations in Loosely Coupled Worlds, Unpublished manuscript, Stanford University.

March, J., y Simon, H. (1969): Les Organizations, Dunod, París. 
MAURICE, M. (1979): «For a study of "The societal effect": Universality and specificity in organization research», en C. Lammers y D. Hickson: 42-60.

Meyer, J., y Rowan, B. (1977): «Institutionalized organizations: Formal Structure as myth and ceremony», en American Journal of Sociology, 83: 340-363, y en M. Zey. Ferrel y M. Aiken (1981): 303-322.

Perrow, C. (1972): Complex Organizations: A Critical Essay, Scott, Foresman, Glenview (I1.).

- (1979): «Demystifying organizations», en SARri, R. C., y Hansenfeld, Y. (eds.): The Management of Human Services, Columbia University Press, Nueva York.

- (1980): "Zoo story" or "Life in the organizational sandpit"», en G. Salaman y K. Thompson: 259-277.

PetTigrew, A. M. (1979): «On studying organizational cultures», en Administrative Science Quarterly, 24: 570-581.

Pfeffer, J. (1982): Organizations and Organization Theory, Pitman, Massachusetts.

Salaman, G., y Thompson, K. (1980): Control and Ideology in Organizations, MIT Press.

Scotт, W. R. (1981): Organizations Rational, Natural and Open Systems, Prentice Hall, Englewood Cliffs (New Jersey).

Sch oenherr, R., y Pérez Vilariño, J. (1979): «Organizational role commitment in the Catholic Church in Spain and the USA», en C. Lammers y D. Hickson: 346-372.

Simon, H. (1962): «The Architecture of Complexity», en Proceedings of the American Pbilosopbical Society, 106: 467-482.

Tannenbaum, A., y Cooke, R. (1979): «Organizational control: a review of studies employing the control graph method», en C. Lammers y D. Hickson: 183-210.

Thompson, J. D. (1967): Organizations in Action, McGraw-Hill, Nueva York.

ThOMPSON, K. (1980): «Organizations as constructors of social reality», en G. Salaman y K. Thompson: 216-256.

VAN DooRN, J. (1979): «Organizations and the social order: A pluralist approach», en C. Lammers y D. Hickson: 61-75.

WEICK, K. (1976): «Educational organizations as loosely coupled systems», en Administrative Science Quarterly, 21: 1-19.

ZALD, M. (1981): «Political Economy: A Framework for comparative analysis», en ZEYFerrel, M., y Aiken, M.: Complex Organizations: Critical Perspectives, Scott Foresman, Glenview (II.). 